\title{
The Robustness of Various Forms of Perimetry to Different Levels of Induced Intraocular Stray Light
}

\author{
Roger S. Anderson, ${ }^{1,2}$ Tony Redmond, ${ }^{1,2}$ D. Rodney McDowell, ${ }^{1}$ Karen M. M. Breslin, ${ }^{1}$ and \\ Margarita B. Zlatkova ${ }^{1}$
}

Purpose. To compare directly the robustness of standard automated perimetry (SAP), short-wavelength automated perimetry (SWAP), frequency-doubling perimetry (FDP), and grating-resolution perimetry (GRP) stimuli to different degrees of intraocular stray light induced by commercially available opacitycontaining filters.

MethoDs. Five white opacity filters of increasing density were used to simulate the typical forward light scatter and stray light values associated with age-related lens opacification and significant cataract. The individually induced intraocular stray light value for each filter was quantified with a stray light meter and plotted against individual perimetric thresholds for the right eyes of three normally sighted trained observers for SAP, SWAP, FDP, and GRP.

Results. All tests were significantly but differently affected by increasing stray light. Overall average declines over a 1 log unit change in the stray light values were as follows: SAP, $4.85 \mathrm{~dB}$; SWAP, $9.03 \mathrm{~dB}$; FDP, $4.29 \mathrm{~dB}$; and GRP, $1.36 \mathrm{~dB}$. Standardized (z) scores were calculated after normalization to the spread of the normative data values for each instrument. These indicated that the standardized changes from baseline over the range of the five filters per log stray light unit were as follows: SAP, 2.177; SWAP, 1.96; FDP, 1.277; and GRP, 1.04.

Conclusions. The increased stray light values induced by cataract-simulating filters has a significant effect on all tests. However, GRP, which is known to be limited by retinal sampling rather than contrast, remains the most robust of the tests to the effects of intraocular stray light. The degree to which the normative "sensitivity" range for different types of perimetry might incorporate a component caused by individual differences in intraocular stray light is discussed and requires further research. (Invest Opbthalmol Vis Sci. 2009;50:4022-4028) DOI:10.1167/iovs.08-2934

$\mathbf{M}$ any types of perimeter tests are available commercially for the purpose of detecting, grading, and monitoring neural damage within the visual pathway. An important requirement of all these tests is to detect neural damage at an early stage. To be able to do this, it is important that the test be

From the ${ }^{1}$ Vision Science Research Group, School of Biomedical Sciences, University of Ulster, Coleraine, Northern Ireland, United Kingdom; and ${ }^{2}$ NIHR Biomedical Research Centre, Moorfields Eye Hospital/UCL Institute of Ophthalmology, London, United Kingdom.

Supported by a PhD Studentship from the Department of Employment and Learning, Northern Ireland, United Kingdom (TR).

Submitted for publication October 1, 2008; revised February 10, 2009; accepted May 12, 2009.

Disclosure: R.S. Anderson, None; T. Redmond, None; D.R. McDowell, None; K.M.M. Breslin, None; M.B. Zlatkova, None

The publication costs of this article were defrayed in part by page charge payment. This article must therefore be marked "advertise$m e n t "$ in accordance with 18 U.S.C. $\$ 1734$ solely to indicate this fact.

Corresponding author: Roger S. Anderson, Vision Science Research Group, School of Biomedical Sciences, University of Ulster, Coleraine, Northern Ireland BT52 1SA, UK; rs.anderson@ulster.ac.uk. as robust as possible to aberrations and other imperfections in the optics of the eye. The need to discriminate between optical and neural visual losses while testing visual function has long been recognized. ${ }^{1-5}$

The effects of optical defocus have been well established for standard automated perimetry (SAP), ${ }^{6-8}$ indicating a significant vulnerability, especially for small stimulus sizes. Shortwavelength automated perimetry (SWAP) has been shown to be resistant to the effect of blur because of the relatively large target size and the poor resolution properties of the SWS pathway. ${ }^{9}$ Frequency-doubling perimetry (FDP) has been found by one study to display significant robustness to the effects of optical blur ${ }^{10}$ and by another to be mildly vulnerable. ${ }^{11}$ The typical low-pass-filtering effects of optical defocus would reasonably be expected to have little effect on a lowfrequency grating stimulus such as that used in FDP $(0.25$ cyc/deg). However, the aging process of the crystalline lens can have a profound effect on retinal image contrast, depending on the spatial frequency and wavelength, as a consequence of increased intraocular stray light and short wavelength light absorption, respectively. ${ }^{12-14}$

Cataract has been shown to have a significant effect on SAP thresholds, ${ }^{15,16}$ though some studies report little effect. ${ }^{17}$ Other studies report that such changes mask glaucomatous change. ${ }^{18-21}$

The effects of intrinsic cataract on SWAP have also been studied, and all those studies report a significant, diffuse effect. Kim et al. ${ }^{22}$ found that cataract predominantly causes a general reduction in sensitivity in both blue-on-yellow and white-onwhite perimetry, but the reduction of blue-on-yellow sensitivity greatly exceeds that of white-on-white sensitivity by 2.4 times, presumably because it is more vulnerable to the effects of age-related lens yellowing. They advocate that caution should be observed when using blue-on-yellow perimetry to evaluate a patient who has lens opacification for glaucoma. Moss et al. ${ }^{23}$ recommend caution in the interpretation of glaucomatous fields in the presence of cataract.

The effect of cataract removal on FDP has been investigated. Tanna et al., ${ }^{24}$ examining patients before and after cataract extraction, found a significant improvement in mean deviation (MD) but not pattern standard deviation (PSD) after surgery. Ueda et al. ${ }^{25}$ reported an improvement in FDP after cataract surgery but no significant difference between eyes with an implant. Casson and James ${ }^{26}$ found a reduction in the false-positive rate postoperatively in otherwise healthy patients, especially for posterior subcapsular cataracts. Siddiqui et al. ${ }^{27}$ found an improvement in MD and a worsening in PSD after cataract surgery in patients with glaucoma, indicating that cataract may mask glaucomatous loss, though other studies $^{24,28,29}$ found no such change in PSD.

These studies examined patients with intrinsic cataract and, as such, did not separate the differing effects of the opacification and yellowing components of lens aging. Different perimetry tests may be affected differently by these separate components of lens aging and cataract. Several studies ${ }^{30-32}$ have used diffusion filters of different density to simulate lens opacity in isolation for SAP and found a generalized reduction in sensitiv- 
ity. Membrey et al. ${ }^{33}$ also found a generalized reduction in sensitivity for FDP when a single diffusing filter was used.

GRP makes use of sinusoidal gratings to test resolution acuity at different locations in the visual field. Previous studies examining grating acuity across the retina have shown that, though foveal acuity is limited by the low-pass-filtering characteristics of the eye's optics, in peripheral vision the lower density sampling array of the retina is often unable to resolve the higher spatial frequencies that pass through the optics (for a review, see Ref. 34). Thus it is possible for the contrast in a grating stimulus to be detectable at peripheral locations, but the subject is unable to resolve the orientation because of its undersampled, aliased appearance. ${ }^{35-39}$ In this way resolution acuity, but not detection acuity, for high-contrast sinusoidal gratings has been reported to be limited by the sampling density of the coarsest array of retinal cells, the ganglion cells, ${ }^{40,41}$ at least in an eye with relatively clear media. However, the effects of different degrees of intraocular light scatter on achromatic grating resolution perimetry remain unknown.

In addition, we have little information about how these perimetric tests are affected relative to each other by agerelated optical imperfections, particularly when those imperfections are of a magnitude that we would associate with normal aging rather than with significant cataract requiring surgery. In the clinical studies, with a few exceptions, visual function was generally investigated in the presence of a fairly gross optical defect (clinically significant cataract). Systems such as the Lens Opacity Classification System (LOCS III), designed to grade cataractous lens appearance according to the degree of yellowing and opacity (observed with a slit lamp), are not suitable to measure more subtle changes in lens scatter associated with normal precataractous aging

One important aspect of lens aging is the increase in stray light at the retina. ${ }^{14}$ Intraocular stray light causes a veiling luminance across the retina in the presence of glare and thus reduces the contrast of the retinal image. Various glare testing methods, typically involving some sort of acuity chart to which a glare source is introduced, have been used to quantify retinal stray light. The repeatability and discriminative power of such tests have been found to be poor. ${ }^{42,43}$ The psychophysical "direct compensation method" proposed by Van den Berg ${ }^{44}$ has shown better sensitivity than the glare testing methods in a wide range of studies ${ }^{45}$ and the recent development of the "compensation comparison" method and its implementation in a commercially available stray light meter (C-Quant; Oculus, Wetzlar, Germany) has permitted us to make more reliable and repeatable measures of intraocular stray light in naive subjects. ${ }^{45,46}$ The compensation comparison technique involves matching the magnitude of flicker in two halves of a central field, one of which has compensation luminance added in counterphase, to a flickering glare source added to the other. ${ }^{45}$ The instrument plots the measured stray light value for each subject against the subject's age on top of a normative age range curve.

In this study we sought to simulate the scatter component of lens aging to different degrees to quantify the extent to which even moderate levels of opacification alone might increase the intraocular stray light value and affect different kinds of perimetry. We can thus investigate the robustness of three perimetric tests, readily available to practitioners, plus the more novel technique of GRP, to increased intraocular stray light induced by simulated lens opacity.

\section{MethodS}

\section{Subjects}

Three experienced psychophysical observers were used in the study: TR (25 years old, -3.00 D myopic, blue irides), RR ( 45 years old, emmetropic, brown irides), and DRM (62 years old, emmetropic, brown irides). All had undergone full eye examination within the previous 3 months and had corrected logMAR visual acuities between -0.14 and -0.10 . Results from a Humphrey visual field test, performed within the previous 3 months, showed normal total deviation and PSD plots for each subject. There was no evidence of any pathologic condition on slit lamp biomicroscopic examination, all lenses were clear of any grossly observable opacification, and there was no evidence of color vision anomalies on Ishihara and City University color vision tests. No subject had undergone any previous ocular surgery. None had any systemic condition or used any medication known to affect visual performance. Intraocular pressures were lower than $22 \mathrm{~mm} \mathrm{Hg}$ in all subjects at the time of recruitment. Rim area parameters were shown by Heidelberg Retinal Tomograph and Moorfields regression analysis to be within normal limits.

Ethical approval for the study was obtained from the Faculty Research Ethics Committee, and the research protocol adhered to the tenets of the Declaration of Helsinki.

\section{Stimuli and Apparatus}

All testing was carried out in the Vision Science laboratory at the University of Ulster (Coleraine, Northern Ireland). The right eyes of the three observers were tested with the Humphrey Visual Field Analyser (HFA; Carl Zeiss Meditec, Dublin, CA) in white-on-white SAP and SWAP modes. Stimuli used were the standard Goldman size III $\left(0.43^{\circ}\right)$ white and Goldman size V $\left(1.73^{\circ}\right)$ blue, respectively. For SWAP, the yellow luminance background was the standard $100 \mathrm{~cd} / \mathrm{m}^{2}$, and the Goldman size $\mathrm{V}$ blue target had a narrow band of peak wavelength $440 \mathrm{~nm}$ and duration of $200 \mathrm{~ms}$.

Subjects also underwent FDP (Welch Allyn, Skaneateles Falls, NY) and a form of GRP. The full-threshold central 24-2 programs for SAP and SWAP were used, as was the full-threshold C20 program for FDP. The FDP stimuli consisted of low spatial frequency $(0.25 \mathrm{cyc} / \mathrm{deg})$ sinusoidal gratings that undergo rapid $(25 \mathrm{~Hz})$ counterphase flicker.

For the GRP test, static achromatic sinusoidal grating patches with the same mean luminance as their surround were generated (Visual Stimulus Generator VSG2/3; Cambridge Research Systems, Rochester, UK) on a 21-inch high-resolution CRT monitor with pixel resolution of $1280 \times 965$ and a frame rate of $73 \mathrm{~Hz}$ (500PS; Sony Corp., Tokyo, Japan). The luminance of the background field was $10 \mathrm{~cd} / \mathrm{m}^{2}$. Chromaticity coordinates for the background and stimuli were $x=0.218$ and $y=0.328$. Gratings were circular patches of $2.5^{\circ}$ diameter and $90 \%$ contrast encased within a Gaussian window and were presented at $20^{\circ}$ eccentricity in each of the four oblique retinal meridians while the subject fixated a central cross. Presentation time was 1 second, including a ramp on-and-off time of 0.3 seconds. Threshold spatial frequency for resolution was determined with a two-alternative forcedchoice staircase psychophysical procedure during which the subject was required to determine the orientation (horizontal or vertical) of the grating by pressing one of two response buttons. Spatial frequency increased by $0.8 \mathrm{~dB}$ after three correct responses and decreased by 0.8 $\mathrm{dB}$ after one incorrect response. Threshold was taken as the mean of the first four reversals.

Each subject was corrected for the working distance of each of the instruments (33 cm for all except FDP), as appropriate, for subject refractive error and age. An appropriate correcting lens for each subject was fitted in the standard lens holder for SAP and SWAP. A wide field spectacle frame was used for FDP and GRP to give maximum field of view, while the fellow eye was occluded with a patch. Pupil diameters were measured before the commencement of each test, with the subject seated in the observation position.

\section{Opacification Filters}

Different degrees of lens opacification were simulated using five white opacity-containing filters (Fog filters; Lee Filters, Andover, UK) to induce light scattering. The opacity filters, described in one of our 
previous papers, ${ }^{47}$ display very flat spectral transmission spectra ranging from 0.86 to 0.42 mean transmittance.

Our previous study also confirmed that the light scattered from a light source at angles between $4^{\circ}$ and $15^{\circ}$ varied inversely proportional to the square of the angular distance, which has been shown to be a typical relationship for the intraocular scatter caused by lens opacification. ${ }^{14}$ With the densest filter, high-contrast logMAR acuity was reduced for each subject by only 0.02 to $0.08 \log$ units, whereas low-contrast (Bailey-Lovie) logMAR acuity was reduced by 0.22 to 0.45 $\log$ units for the same filter.

In addition, we used a stray light meter (C-Quant; Oculus) to measure the baseline intraocular stray light for each subject and the individual increase in intraocular stray light that resulted when each of the five filters was placed in front of the eye close to the cornea. The stray light meter plot in Figure 1a shows the baseline measurements for subjects (black dots) plotted against their ages. Subject TR displays above average stray light for age and subject DRM displays below average stray light for age, but all values are within the normal range indicated by the gray band.

Figure 1b shows how the stray light value for the 45-year-old subject (RR) changed for each filter. It can be seen that the filter (Fog 1; Lee Filters) effectively affords an average 45-year-old the stray light value of an average 58-year-old. Filters 2 and 3 (Fog 1; Lee Filters) turn the subject into an average 72 - and 82 -year-old, respectively (i.e., each filter adds an extra 10 to 14 years to the subject's stray light value). Filters 4 and 5 take the subject into the realm of significant cataract. The effect was similar for the other two subjects. By way of comparison, the gray dot represents a 64 -year-old patient with cataract dis-
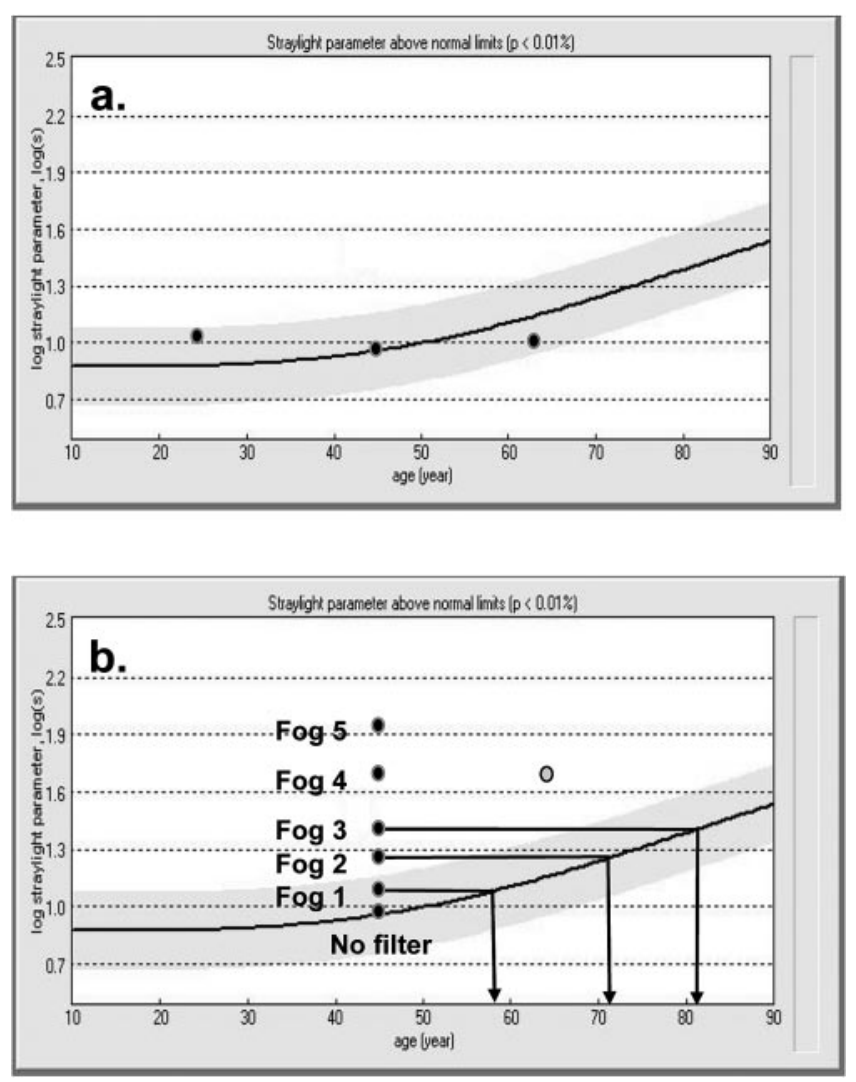

FiguRE 1. (a) Baseline stray light value for each of the three subjects measured with the stray light meter. All values were within normal range for subject age. (b) Stray light value for the 45-year-old subject (RR) for each filter. Filters 1 to 3 successively add approximately 15 years to the subject's baseline stray light measurement. Filters 4 and 5 take the subject into the realms of significant cataract. Gray dot: 64-year-old patient with good acuity and low LOCSIII score but awaiting cataract surgery because of increased stray light value. playing reasonable visual acuity (0.12 logMAR) and LOCSIII grading (NO2, C3, P1) but awaiting surgery in the hospital based on his stray light value (1.7).

The stray light meter (C-Quant; Oculus) thus yields a clinically meaningful indication of the degree to which each filter increases the forward light scatter for each subject, and the perimetric threshold can then be plotted against the actual stray light value for each filter condition. For analysis, the stimulus locations closest to those of the GRP test $\left(20^{\circ}\right.$ eccentricity in the four oblique meridians) were extracted from the SAP, SWAP, and FDT plots. For each test condition, it was noted whether any of these locations fell outside the $95 \%$ confidence limit of the instrument.

During each testing session there was at least a 5-minute interval between each test to overcome fatigue, and observers were allowed a minimum of 2 minutes' adaptation time to the yellow background before beginning each SWAP test.

\section{Results}

All visual field plot results, for all observers, were within acceptable levels of reliability ( $<20 \% \mathrm{FP},<33 \% \mathrm{FN},<33 \% \mathrm{FL})$. Perimetric thresholds plotted against the induced stray light values for each subject can be seen in Figure 2. For initial comparison, all thresholds were expressed in the standard perimetry units of decibels $(\mathrm{dB} ; 1 \mathrm{~dB}=0.1 \log$ unit change). (It should be noted that, for the FDT, contrast is normally based on stimulus amplitude rather than power, which means that 1 $\mathrm{dB}=0.05 \log$ unit; however we have here converted all threshold $\mathrm{dB}$ units to $0.1 \log$ threshold change). For GRP, thresholds were converted from cyc/deg so that $1 \mathrm{~dB}$ represents a $0.1 \mathrm{log}$ unit change from a $1 \mathrm{cyc} / \mathrm{deg}$ reference level. The decibel scale has been kept fixed for each plot (20-dB range). Looking at the overall sensitivities (heights of the curves), subject RR (an emmetrope with dark eyes) displayed highest sensitivity for SWAP and FDP. Subject TR (a lowmedium myope with blue eyes), despite being the youngest subject, had an overall sensitivity comparable to that of the other, older, emmetropic subjects for GRP but lower than that of the others for SAP, SWAP, and FDP.

Given that these differences in the heights of the curves represented real between-subject differences in overall sensitivity, rather than measurement variability, we performed repeated-measures ANOVA in which the subject differences were removed from the error estimate to isolate the effect of stray light. All tests were progressively and significantly affected by increasing stray light values $(P<0.05)$ but to different degrees. SAP demonstrated an overall average decline of $4.85 \mathrm{~dB} / \log$ stray light unit $(P<0.001)$, SWAP $9.03 \mathrm{~dB} / \log$ stray light unit $(P<0.001)$, FDP $8.06 \mathrm{~dB} / \log$ stray light unit $(P<0.001)$, and GRP $1.36 \mathrm{~dB} / \log$ stray light unit $(P=0.014)$ over the range of five filter values. Fisher LSD post hoc analysis indicated that the mean sensitivity values became significantly different $(P<0.05)$ from the baseline at the third filter for SAP, the second filter for SWAP, and the first filter for FDP but not until the fourth filter for GRP. The filled symbols on each plot indicate a test condition in which one or more of the four analyzed locations was indicated by the instrument to fall outside the " $2 \%$ probability" limit (from StatPac for Windows, available at http://www.statpac.com), i.e., close to the lower limit of the $2.5 \%$ to $97.5 \%$ (95\%) confidence range.

However, a threshold change of $1 \mathrm{~dB}$ (or $0.1 \mathrm{log}$ unit) for one instrument is not necessarily the same as a 1-dB change for another instrument. SAP and SWAP measures $\Delta I / I$ (Weber) contrast, FDP uses Michelson contrast, and GRP measures threshold spatial frequency. To yield a more clinically meaningful comparison, the normative 95\% threshold confidence ranges were separately derived from previous studies for 
Sensitivity (dB)

Sensitivity

(dB)

Sensitivity (dB)

Figure 2. Perimetric sensitivity/ resolution $(\mathrm{dB})$ versus log stray light value for (a) SAP, (b) SWAP, (c) FDP, and (d) GRP for all three subjects. For all perimeters, $1 \mathrm{~dB}$ here represents $0.1 \log$ unit change in threshold.

Resolution (dB)
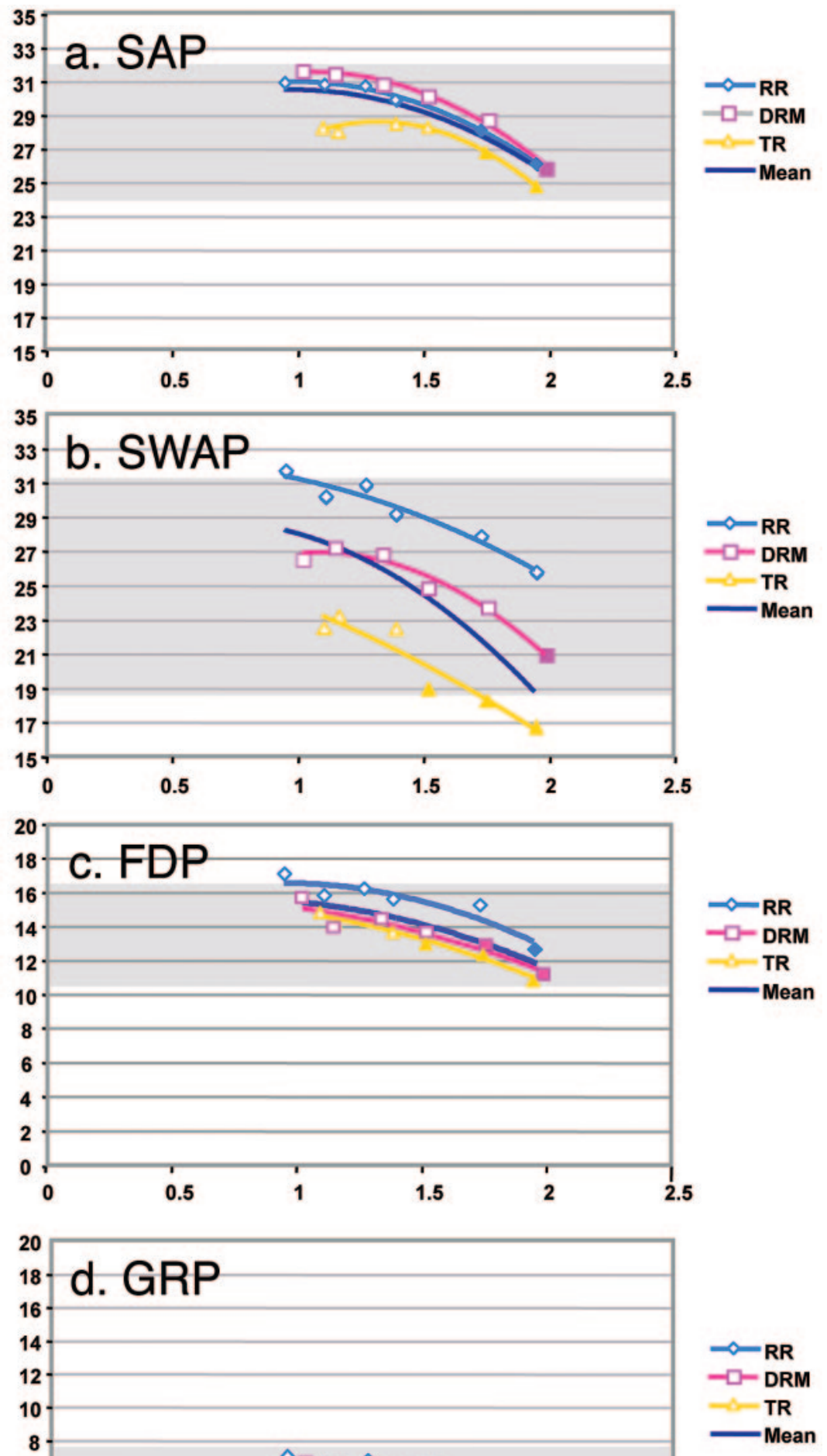

SAP, ${ }^{48}$ SWAP, ${ }^{48,49} \mathrm{FDT}^{50},{ }^{50}$ and $\mathrm{GRP}{ }^{51}$ at this eccentricity and for this age range and are indicated by the gray bands on each plot. For SAP, despite a significant change in the averaged $\mathrm{dB}$ value, all points remained within the $95 \%$ confidence range for the test, in part because the subjects' baseline sensitivities lie in the upper region of the normative range. The final stray light value for subject DRM and the final two stray light values for subjects TR and RR (filled symbols) resulted in at least one of the four 
points being highlighted by the instrument as falling outside the $2 \%$ probability. For SWAP, there was a greater range of baseline sensitivities, and only one subject (TR, with myopia) strayed outside the normative sensitivity range (at the two highest stray light values); the instrument also indicated at least one point as abnormal at $2 \%$ probability at these stray light values. For FDP, all subjects began with high baseline sensitivities and remained within the normative range. However, the instrument indicated at least one abnormal point at the higher stray light values. For GRP, all subjects remained within the normative range at all stray light values, and no individual locations fell outside the $95 \%$ probability range at any time.

We also compared the effects of stray light on different perimeters by using $z$-scores, based on the normal data for each perimeter. First we normalized the baseline threshold values to the mean of the normative data ranges for each instrument. This allowed us to compute the standardized $(z)$ scores for the change from baseline for each subject using the SD of the normative range. Over the range of the five filters, the decline in standardized score from baseline was 2.177 U/U log stray light for SAP, $1.96 \mathrm{U} / \mathrm{U} \log$ stray light for SWAP, $1.277 \mathrm{U} / \mathrm{U} \log$ stray light for FDP, and $1.04 \mathrm{U} / \mathrm{U} \log$ stray light for GRP.

\section{Discussion}

When testing patients for visual damage, it is important that any test used be capable of differentiating neural and optical losses of vision. Perimetry has as its goal the detection, grading, and monitoring of neural loss in the visual pathway and, as such, should be as robust as possible to the effects of optical imperfections be they optical aberrations, age-related lens yellowing, or opacification. Previous studies have shown declining performance with age for SAP,${ }^{52,53}$ FDP,${ }^{50,54}$ GRP,${ }^{51,55}$ and especially SWAP ${ }^{49,54}$ which has also been found to display larger interindividual variability with age. ${ }^{48}$ However, it is not always easy to determine what proportion of this age-related decline is attributed to optical rather than neural change for each perimetry type. The separate contribution of different optical factors to perimetric sensitivity loss is not easily discernible in the intrinsic form, particularly for moderate levels of age-related filtering, which may not be readily observed or categorized when using standard clinical observation methods. For this reason, we sought to induce different levels of opacification to determine the extent to which different individual perimetric thresholds are affected by the resultant increase in intraocular stray light, without the confounding effects of interindividual variations in aberration, lens yellowing, or neural sensitivity.

As discussed, subject TR, the only blue-eyed subject, consistently displayed lower sensitivity than the other two emmetropic subjects, especially for SAP and SWAP. In addition to scatter from the cornea and lens, intraocular stray light has been shown to have components arising from passage of light through the iris and scleral wall and subsequent fundus reflectance, ${ }^{56,57}$ all largely dependent on ocular pigmentation. The overall difference observed in the height of the curve for subject TR may also, in large part, be explained by the fact that he is the only myope. Again, our overall sensitivity findings are largely in accordance with previous studies that report lower sensitivity in myopes for $\mathrm{SAP}^{58,59}$ regardless of the method of optical correction, ${ }^{60}$ SWAP $^{61}$ and GRP ${ }^{62}$ but not for FDP. ${ }^{63}$

Simulated lens opacification has a significant effect (in decibel terms) on all tests but to a lesser extent for GRP. Diffusing filters have previously been shown to affect performance for SAP and FDP ${ }^{33}$ FDP, which measures contrast sensitivity using low spatial frequency phase-reversing grating, may not be significantly affected by the low-pass-filtering effects of optical blur ${ }^{10}$ but suffers significantly at the hands of wide-angle light scatter of even mild levels. The result for GRP builds on previous laboratory studies that have shown grating resolution in the periphery is limited by retinal ganglion cell sampling density. This sampling, rather than optical, limit to performance has been shown to allow peripheral resolution acuity to remain robust to optical defocus up to $3 \mathrm{D}^{4,5}$ and to display no change in performance when grating contrast is reduced to as low as $20 \%{ }^{64}$ The same appears to be true for wide-angle scatter.

Although GRP appears to be more robust to the effects of forward light scatter than the other tests in decibel terms, the determination of clinically meaningful change for each type of test is still not straightforward. Any change in sensitivity resulting from increasing light scatter would have to take the patient outside the limits of the normative sensitivity value to be interpreted by the instrument as clinically significant. The problem with determining what constitutes a clinically meaningful change in this way is that the normative sensitivity range for each test includes, in different proportions, a neural and an optical component. It can be seen in Figure 2 that the normative range of sensitivity values is different for each of the perimetry tests used here, but less understood is the degree to which the variability in the instrument normative databases represents individual variations in intraocular stray light and true neural sensitivity differences. A perimetry test that displays poor robustness (in decibel terms) to increasing intraocular stray light may reasonably be expected to demonstrate a confoundingly greater variation in the normative range. This is borne out to some degree in the present data: in terms of decibels, SWAP displays a greater vulnerability than SAP to stray light but also a greater normative sensitivity range. Because of this larger normative variation (possibly as a result of greater scatter effects for short-wavelength stimuli), differences in the $z$-scores are smaller for SWAP than for SAP. The $z$-score thus masks the true vulnerability of SWAP to increased stray light. The same may be true for FDP. GRP displays the lowest vulnerability to intraocular stray light in decibels but also the narrowest normative threshold range, possibly because the interindividual scatter/stray light component of variability (which is retinal sampling limited rather than contrast limited) is lower. Although this means the $z$-score is not flattered by a large SD to the same degree as the other tests, it implies that any change observed in threshold, such as during testing for glaucoma, is more likely to represent true neural loss rather than optical attenuation.

Further work is required to better identify the components of perimetric sensitivity that are of optical rather than neural origin. The authors suggest that the term sensitivity be used more thoughtfully in the field of perimetry and that it be used primarily when we are confident that we are observing change in neural function rather than optical vulnerability.

Clinical practice should strive to make use of stimuli that better separate optical and neural losses of vision to better detect and monitor diseases that cause damage to the neural visual pathway.

\section{References}

1. Thibos LN, Bradley A. New methods for discriminating neural and optical losses of vision. Optom Vis Sci. 1993;70(4):279-287.

2. Elliott DB. Contrast sensitivity decline with aging: a neural or optical phenomenon. Opbthal Physiol Opt. 1987;7(4):415-419.

3. Morrison JD, McGrath C. Assessment of the optical contributions to age-related deterioration in vision. Quart J Exp Physiol. 1985; $70(2): 249-269$

4. Anderson RS. The selective effect of optical defocus on detection and resolution acuity in peripheral vision. Curr Eye Res. 1996; 15(3):351-353.

5. Wang YZ, Thibos LN, Bradley A. Effects of refractive error on detection acuity and resolution acuity in peripheral vision. Invest Opbthalmol Vis Sci. 1997;38(10):2134-2143. 
6. Heuer DK, Anderson DR, Feuer WJ, Gressel MG. The influence of refraction accuracy on automated perimetric threshold measurements. Ophthalmology. 1987;94(12):1550-1553.

7. Herse PR. Factors influencing normal perimetric thresholds obtained using the Humphrey Field Analyzer. Invest Ophthalmol Vis Sci. 1992;33(3):611-617.

8. Weinreb RN, Perlman JP. The effect of refractive correction on automated perimetric thresholds. Am J Ophthalmol. 1986;101(6):706709.

9. Johnson CA, Adams AJ, Casson EJ. Blue-on-yellow perimetry: a five year overview. In: Mills RP, ed. Perimetry Update 1992/93. Amsterdam: Kugler Publications. 1993:459- 466.

10. Anderson AJ, Johnson CA. Frequency-doubling technology perimetry and optical defocus. Invest Ophthalmol Vis Sci. 2003;44(9): 4147-4152.

11. Artes PH, Nicolela MT, McCormick TA, LeBlanc RP, Chauhan BC. Effects of blur and repeated testing on sensitivity estimates with frequency doubling perimetry. Invest Ophthalmol Vis Sci. 2003; 44(2):646-652.

12. Weale RA. New light on old eyes. Nature. 1963;198:944-946.

13. Wolf E Gardiner IS. Studies on the scatter of light in the dioptric media of the eye as a basis of visual glare. Arch Ophthalmol. 1965;74:338-345.

14. Ijspeert JK, de Waard PW, van den Berg TJ, de Jong PT. The intraocular stray light function in 129 healthy volunteers: dependence on angle, age and pigmentation. Vision Res. 1990;30(5):699-707.

15. Lam BL, Alward WL, Kolder HE. Effect of cataract on automated perimetry. Ophthalmology. 1991;98(7):1066-1070.

16. Klein BE, Klein R, Jensen SC. Visual sensitivity and age-related eye diseases: the Beaver Dam Eye Study. Ophthalmic Epidemiol. 1996; 3(1):47-55.

17. Stewart WC, Rogers GM, Crinkley CM, Carlson AN. Effect of cataract extraction on automated fields in chronic open-angle glaucoma. Arch Ophthalmol. 1995;113(7):875-879.

18. Heider HW, Seez KJ, Schnaudigel OE. Changes in the visual field caused by lens opacities. Klin Monatsbl Augenbeilkd. 1991; 198(1):15-19.

19. Smith SD, Katz J, Quigley HA. Effect of cataract extraction on the results of automated perimetry in glaucoma. Arch Ophthalmol. 1997;115(12):1515-1519.

20. Bengtsson B, Lindgren A, Heijl A, Lindgren G, Asman P, Patella M. Perimetric probability maps to separate change caused by glaucoma from that caused by cataract. Acta Ophthalmol Scand. 1997; 75(2): $184-188$.

21. Hayashi K, Hayashi H, Nakao F, Hayashi F. Influence of cataract surgery on automated perimetry in patients with glaucoma. Am J Ophthalmol. 2001;132(1):41-46.

22. Kim YY, Kim JS, Shin DH, Kim C, Jung HR. Effect of cataract extraction on blue-on-yellow visual field. Am J Ophthalmol. 2001; 132(2):217-220.

23. Moss ID, Wild JM, Whitaker DJ. The influence of age-related cataract on blue-on-yellow perimetry. Invest Ophthalmol Vis Sci. 1995;36(5):764-773.

24. Tanna AP, Abraham C, Lai J, Shen J. Impact of cataract on the results of frequency-doubling technology perimetry. Ophthalmology. 2004;111(8):1504-1507.

25. Ueda T, Ota T, Yukawa E, Hara Y. Frequency doubling technology perimetry after clear and yellow intraocular lens implantation. Am J Ophthalmol. 2006;142(5):856-858.

26. Casson RJ, James B. Effect of cataract on frequency doubling perimetry in the screening mode. J Glaucoma. 2006;15(1):23-25.

27. Siddiqui MA, Azuara-Blanco A, Neville S. Effect of cataract extraction on frequency doubling technology perimetry in patients with glaucoma. Br J Ophthalmol. 2005;89(12):1569-1571.

28. Kook MS, Yang SJ, Kim S, Chung J, Kim ST, Tchah H. Effect of cataract extraction on frequency doubling technology perimetry. Am J Ophthalmol. 2004;138(1):85-90.

29. Arvind H, George R, Baskaran M, et al. Effect of cataract surgery with intraocular lens implant on frequency doubling perimetry (published correction appears in Curr Eye Res. 2005;30(11): 1033). Curr Eye Res. 2005;30(2):123-128.
30. Heuer DK, Anderson DR, Knighton RW, Feuer WJ, Gressel MG. The influence of simulated light scattering on automated perimetric threshold measurements. Arch Ophthalmol. 1988;106(9):1247-1251.

31. Budenz DL, Feuer WJ, Anderson DR. The effect of simulated cataract on the glaucomatous visual field. Ophthalmology. 1993; 100(4):511-517.

32. Uyama K, Matsumoto C, Okuyama S, Otori T. The influence of target blurring and simulated opacity of the ocular media on automated perimetric thresholds. Nippon Ganka Gakkai Zasshi. 1993;97(8):994-1001.

33. Membrey L, Kogure S, Fitzke FW. A comparison of the effects of neutral density filters and diffusing filters on motion detection perimetry, white-on-white perimetry and frequency doubling perimetry. In: Wall M, Wild JM, eds. Perimetry Update 1998/99. Amsterdam: Kugler Publications; 1999:75-83.

34. Anderson RS. The psychophysics of glaucoma: improving the structure/function relationship. Prog Ret Eye Res. 2006;25:79-97.

35. Williams DR, Collier R. Consequences of spatial sampling by a human photoreceptor mosaic. Science. 1983;221(4608):385-387.

36. Smith RA, Cass PF. Aliasing in the parafovea with incoherent light. J Opt Soc Am A. 1987;4(8):1530 - 1534.

37. Thibos LN, Walsh DJ, Cheney FE. Vision beyond the resolution limit: aliasing in the periphery. Vision Res. 1987;27(12):2193-2197.

38. Anderson SJ, Hess RF. Post-receptoral undersampling in normal human peripheral vision. Vision Res. 1990;30(10):1507-1515.

39. Williams DR, Artal P, Navarro R, McMahon MJ, Brainard DH. Off-axis optical quality and retinal sampling in the human eye. Vision Res. 1996;36(8):1103-1114.

40. Thibos LN, Cheney FE, Walsh DJ. Retinal limits to the detection and resolution of gratings. J Opt Soc Am A. 1987;4(8):1524-1529.

41. Anderson RS, Zlatkova MB, Demirel S. What limits detection and resolution of short-wavelength sinusoidal gratings across the retina? Vision Res. 2002;42(8):981-990.

42. Elliott DB, Bullimore MA. Assessing the reliability, discriminative ability and validity of disability glare tests. Invest Ophthalmol Vis Sci. 1993;34:108-119.

43. Van Rijn LJ, Nischler C, Gamer D, et al. Measurement of stray light and glare: comparison of Nyktotest, Mesotest, stray light meter and computer implemented stray light meter. Br J Ophthalmol. 2005; 89:345-351.

44. Van den Berg TJTP. Importance of pathological intraocular light scatter for visual disability. Doc Ophthalmol. 1986;61:327-333.

45. Franssen L, Coppens JE, van den Berg TJ. Compensation comparison method for assessment of retinal stray light. Invest Ophthalmol Vis Sci. 2006;47(2):768-776.

46. Franssen L, Coppens JE, van den Berg TJ. Modulation depth threshold in the compensation comparison approach. $J$ Vision. 2007; 7(1):8;1-14.

47. Zlatkova MB, Coulter EE, Anderson RS. The effect of simulated lens yellowing and opacification on blue-on-yellow acuity and contrast sensitivity. Vision Res. 2006;46(15):2432-2442.

48. Wild JM, Cubbidge RP, Pacey IE, Robinson R. Statistical aspects of the normal visual field in short-wavelength automated perimetry. Invest Ophthalmol Vis Sci. 1998;39(1):54-63.

49. Bengtsson B, Heijl A. Normal intersubject threshold variability and normal limits of the SITA SWAP and full threshold SWAP perimetric programs. Invest Ophthalmol Vis Sci. 2003;44(11):5029-5034.

50. Anderson AJ, Johnson CA, Fingeret M, et al. Characteristics of the normative database for the Humphrey matrix perimeter. Invest Ophthalmol Vis Sci. 2005;46(4):1540-1548.

51. Anderson RS, McDowell DR. Peripheral resolution using stationary and flickering gratings: the effects of age. Curr Eye Res. 1997; 16(12):1209-1214.

52. Heijl A, Lindgren G, Olsson J. Perimetric threshold variability and age. Arch Ophthalmol. 1988;106(4):450 - 452.

53. Hermann A, Paetzold J, Vonthein R, Krapp E, Rauscher S, Schiefer U. Age-dependent normative values for differential luminance sensitivity in automated static perimetry using the Octopus 101. Acta Ophthalmol Scand. 2008;86(4):446-455.

54. Gardiner SK, Johnson CA, Spry PG. Normal age-related sensitivity loss for a variety of visual functions throughout the visual field. Optom Vis Sci. 2006;83(7):438-443. 
55. Zlatkova MB, Coulter E, Anderson RS. Short-wavelength acuity: blue-yellow and achromatic resolution loss with age. Vision Res. 2003;43(1):109-115.

56. Van den Berg TJ, Ijspeert JK, de Waard PW. Dependence of intraocular straylight on pigmentation and light transmission through the ocular wall. Vision Res. 1991;31(7-8):1361-1367.

57. Coppens JE, Franssen L, van den Berg TJ. Wavelength dependence of intraocular straylight. Exp Eye Res. 2006;82(4):688-692.

58. Rudnicka AR, Edgar DF. Automated static perimetry in myopes with peripapillary crescents-Part II. Opbthalmic Pbysiol Opt. 1996;16(5):416-429.

59. Nitta K, Saito Y, Sugiyama K. The influence on the static visual field of peripapillary chorioretinal atrophy-relation to refractive error [in Japanese]. Nippon Ganka Gakkai Zassbi. 2006;110(9):693697.
60. Aung T, Foster PJ, Seah SK, et al. Automated static perimetry: the influence of myopia and its method of correction. Opbthalmol. 2001;108(2):290-295.

61. Kawabata H, Fujimoto N, Adachi-Usami E. Sensitivity loss of short wavelength sensitive cones in myopic eyes by blue-on-yellow perimetry [in Japanese]. Nippon Ganka Gakkai Zasshi. 1997; 101(8);648-655.

62. Chui TY, Yap MK, Chan HH, Thibos LN. Retinal stretching limits peripheral visual acuity in myopia. Vision Res. 2005;45(5):593-605.

63. Ito A, Kawabata H, Fujimoto N, Adachi-Usami E. Effect of myopia on frequency-doubling perimetry. Invest Opbthalmol Vis Sci. 2001;42(5):1107-1110.

64. Thibos LN, Still DL, Bradley A. Characterization of spatial aliasing and contrast sensitivity in peripheral vision. Vision Res. 1996; 36(2):249-258. 